INPLASY

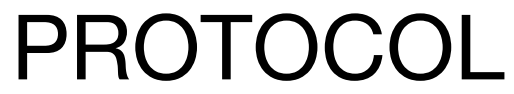

To cite: Yang et al. Effect of beta-blocker therapy on mortality after colorectal cancer surgery: a metaanalysis. Inplasy protocol 2020100092. doi:

10.37766/inplasy2020.10.0092

Received: 24 October 2020

Published: 24 October 2020

Corresponding author:

Guang-Zhu Su

suguangzhu@126.com

Author Affiliation:

Central Hospital Affiliated

Shandong First Medical

University

Support: None.

Review Stage at time of this submission: The review has not yet started.

Conflicts of interest:

None.

\section{Effect of beta-blocker therapy on mortality after colorectal cancer} surgery: a meta-analysis

\author{
Yang, $\mathrm{HY}^{1}$; Sun, ML2; Su, GZ³.
}

Review question / Objective: Is beta-blocker therapy associated with a reduction in mortality after colorectal cancer surgery?

Condition being studied: Colorectal cancer surgery.

Information sources: Studies were identified by searching electronic databases including PubMed and Embase. No limits were applied. We used controlled vocabulary (MeSH in PubMed and Emtree in Embase) and keywords as search terms. In addition, we hand searched the reference lists of eligible studies.

INPLASY registration number: This protocol was registered with the International Platform of Registered Systematic Review and Meta-Analysis Protocols (INPLASY) on 24 October 2020 and was last updated on 24 October 2020 (registration number INPLASY2020100092).

\section{INTRODUCTION}

Review question / Objective: Is betablocker therapy associated with a reduction in mortality after colorectal cancer surgery?
Rationale: Colorectal cancer surgery remains associated with substantial postoperative morbidity and mortality despite advances in surgical techniques and care. Induced adrenergic hyperactivity is thought to be an important contributor. 
Beta-blocker therapy may be linked to improved postoperative outcomes.

Condition being studied: Colorectal cancer surgery.

\section{METHODS}

Search strategy: ( $\beta$ block*[ti] OR beta

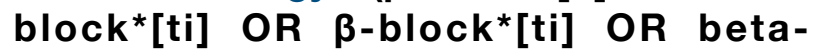
block $\left.^{\star}[\mathrm{ti}]\right)$ (colon[ti] OR colonic[ti] OR rectal[ti] OR colorectal[ti]).

Participant or population: Patients undergoing colorectal cancer surgery.

Intervention: Beta-blocker therapy.

Comparator: No beta-blocker therapy.

Study designs to be included: Cohort studies.

Eligibility criteria: Cohort studies that investigated the association between betablocker therapy and mortality after colorectal cancer surgery.

Information sources: Studies were identified by searching electronic databases including PubMed and Embase. No limits were applied. We used controlled vocabulary (MeSH in PubMed and Emtree in Embase) and keywords as search terms. In addition, we hand searched the reference lists of eligible studies.

Main outcome(s): 1-year all-cause mortality.

Additional outcome(s): Complications and other timepoint mortality.

Data management: We developed a data extraction sheet in standardized Excel (Microsoft Corporation).

Quality assessment / Risk of bias analysis: We evaluated the quality of included studies by using the Newcastle-Ottawa Scale, which is a scale for assessing the quality of observational studies in metaanalyses.
Strategy of data synthesis: The metaanalyses were performed by computing odds ratios (ORs) with $95 \%$ Cls for mortality using a random-effects model, accounting for clinical heterogeneity.

Subgroup analysis: None.

Sensibility analysis: None.

Country(ies) involved: China.

Keywords: Colorectal cancer surgery; betablocker; mortality.

Contributions of each author:

Author 1 - Hong-Yan Yang.

Author 2 - Mei-Li Sun.

Author 3 - Guang-Zhu Su. 